\title{
Dynamical Correlations near Dislocation Jamming
}

\author{
Lasse Laurson, ${ }^{1}$ M.-Carmen Miguel, ${ }^{2}$ and Mikko J. Alava ${ }^{3}$ \\ ${ }^{1}$ ISI Foundation, Viale S. Severo 65, 10133 Torino, Italy \\ ${ }^{2}$ Departament de Física Fonamental, Facultat de Física, Universitat de Barcelona, Diagonal 647, 08028 Barcelona, Spain \\ ${ }^{3}$ Department of Applied Physics, Aalto University, P.O. Box 14100, 00076 Aalto, Espoo, Finland
}

(Received 11 February 2010; published 28 June 2010)

\begin{abstract}
Dislocation assemblies exhibit a jamming or yielding transition at a critical external shear stress value $\sigma=\sigma_{c}$. Here we study the heterogeneous and collective nature of dislocation dynamics within a crystal plasticity model close to $\sigma_{c}$, by considering the first-passage properties of the dislocation dynamics. As the transition is approached in the moving phase, the first-passage time distribution exhibits scaling, and a related peak dynamical susceptibility $\chi_{4}^{*}$ diverges as $\chi_{4}^{*} \sim\left(\sigma-\sigma_{c}\right)^{-\alpha}$, with $\alpha \approx 1$.1. We relate this scaling to an avalanche description of the dynamics. While the static structural correlations are found to be independent of the external stress, we identify a diverging dynamical correlation length $\xi_{y}$ in the direction perpendicular to the dislocation glide motion.
\end{abstract}

DOI: 10.1103/PhysRevLett.105.015501

PACS numbers: 61.72.Lk, 68.35.Rh, 81.40.Lm

The mechanical behavior of crystalline solids subject to an external shear stress $\sigma$ is controlled by a finite yield stress $\sigma_{c}$ : For stresses below it, only elastic or reversible deformation of the material takes place, while sustained plastic or irreversible deformation mediated by dislocation motion is observed for $\sigma>\sigma_{c}$. The basic phenomenology of two states with different rheology is similar also for many amorphous materials ranging from foams to amorphous and granular media, even though the microscopic mechanisms responsible for a transition to "flow" are not yet well understood. The concept of jamming has been introduced to comprehend the observed phenomenology: In a finite region of the parameter space (spanned by control variables such as temperature, density, and an external force), due to a "cage effect," or self-induced constraints on the motion of the system constituent particles, the phase space dynamics gets restricted, i.e., jammed [1-4]. For two-dimensional ensembles of crystal dislocations, a yielding transition at a finite $\sigma_{c}$ has been established, even in the absence of any external sources of disorder [5]. The crucial mechanism here is the generation of effective disorder in the local stress field, due to constrained dislocation motion by the combined effect of the slip geometry and the long-range anisotropic dislocationdislocation interactions. These are also manifest in the formation of metastable structures such as dislocation dipoles and walls.

On approaching the jamming transition from the moving phase, the dynamics generally becomes increasingly heterogeneous. A growing dynamical correlation length characterizing spatial and temporal correlations has been observed for granular systems [6,7], as well as on approaching the glass transition of molecular liquids and colloidal suspensions [8]. In this work, we consider dislocation jamming in a similar vein. Known features of the 2D discrete dislocation dynamics (DDD) model studied here are a relaxation of the strain rate or an Andrade law: Close to $\sigma=\sigma_{c}$, it follows a power law $\dot{\boldsymbol{\epsilon}} \sim t^{-\theta}, \theta \approx 2 / 3$ [5]. The steady state strain rate displays a nonlinear rheological behavior, $\dot{\epsilon} \sim\left(\sigma-\sigma_{c}\right)^{\beta}$, with $\beta \approx 1.8$ [5]. The model also reproduces the experimentally observed scalefree size distributions of avalanches of plastic deformation [9]. Similar DDD models are used also in many materials science applications [10]. The question is now whether one can establish the jamming or yielding transition as a second-order phase transition. This would imply the existence of a length scale, the correlation length, diverging at the transition point. The dislocation system nonequilibrium transition is particular, since the temperature is included only indirectly via the dislocation mobility and is thus irrelevant.

The avalanches exhibited by dislocation assemblies [9] are suitable candidates for fundamental "dynamical heterogeneities" or localized events. We define appropriate statistical quantities to characterize the dynamics via timedependent first-passage probabilities, which measure the likelihood that a dislocation becomes liberated from the confining stress field over an observation scale. The firstpassage distributions are found to display scaling close to the transition, and, analogously to other jamming systems, the peak value of a dynamical susceptibility and a dynamical correlation length grow on approach and diverge at $\sigma=\sigma_{c}$. At the same time, static correlations describing the dislocation structures are found to be virtually independent of the external stress. We also connect the results to an avalanche description of the dynamics.

The DDD model represents a 2D cross section ( $x y$ plane) of a 3D single crystal with a single slip geometry $[5,9,11]$. The dislocations are straight parallel edge ones, with the dislocation lines along the $z$ axis. The $N$ pointlike dislocations glide along directions parallel to their Burgers vectors $\vec{b}_{n}=s_{n} b \vec{u}_{x}$, with $s_{n}= \pm 1$ and $\vec{u}_{x}$ the unit vector in the 
$x$ direction. Equal numbers of dislocations with positive and negative Burgers vectors are considered. Dislocation climb is excluded for simplicity. The dislocations interact with each other through their anisotropic long-range stress fields, $\quad \sigma_{s}(\vec{r})=D b x\left(x^{2}-y^{2}\right) /\left(x^{2}+y^{2}\right)^{2}$, where $D=$ $\mu / 2 \pi(1-\nu)$, with $\mu$ the shear modulus and $\nu$ the material's Poisson ratio. The dynamics is taken to be overdamped with a linear force-velocity relation, with the equations of motion

$$
\frac{\chi_{d}^{-1} v_{n}}{b}=s_{n} b\left[\sum_{m \neq n} s_{m} \sigma_{s}\left(\vec{r}_{n m}\right)+\sigma\right],
$$

where $v_{n}$ is the velocity of the $n$th dislocation, $\chi_{d}$ is the dislocation mobility, $s_{n}$ is the sign of the Burgers vector of the $n$th dislocation, and $\sigma$ is the applied external shear stress. The long-range interaction forces are computed by imposing periodic boundary conditions in both $x$ and $y$ directions. The equations of motion are integrated numerically with an adaptive step size fifth-order Runge-Kutta algorithm in dimensionless units by measuring lengths in units of $b$, times in units of $1 /\left(\chi_{d} D b\right)$, and stresses in units of $D$. If the distance between two dislocations of opposite sign gets smaller than $2 b$, they are removed, a process reminiscent of the annihilations occurring in real crystals. The simulations are started from a random initial configuration of $N_{0}$ dislocations within a square cell of linear size $L$. The system is first let to relax with $\sigma=0$, until it reaches a metastable dislocation arrangement. During this relaxation, a significant fraction gets annihilated. We take $L=200 b$ and $300 b$, with $N_{0}=1600$ and 3600, respectively. After the relaxation, the external stress is switched on, and the evolution of the system is monitored. For $\sigma>\sigma_{c} \approx 0.025$ [12], the system eventually reaches a steady state, with the strain rate $\dot{\epsilon} \sim \sum_{n} b_{n} v_{n}$ fluctuating around a constant mean value. The steady state consists of $N \approx 375$ dislocations for $L=200 b$ and $N \approx 850$ for $L=$ $300 b$ [13].

To characterize the heterogeneous dynamics we first consider the first-passage time $\left(t_{\mathrm{fp}}\right)$ distribution of dislocations in the moving steady state. $t_{\mathrm{fp}}$ is defined as the time at which a dislocation first moves across a distance $l$ from some initial position and thus becomes liberated from a confining stress field at this scale. Given the nonlinear stress dependence of the steady state strain rate [5], the mean first-passage time is expected to behave like $\left\langle t_{\mathrm{fp}}\right\rangle \sim$ $l /\langle v\rangle \sim l\left(\sigma-\sigma_{c}\right)^{-\beta}$. Assuming $\delta t_{\mathrm{fp}} \sim\left\langle t_{\mathrm{fp}}\right\rangle$, one can write

$$
P\left(t_{\mathrm{fp}} ; l, \sigma\right)=\frac{1}{l\left(\sigma-\sigma_{c}\right)^{-\beta}} \mathcal{F}\left[\frac{t_{\mathrm{fp}}-l\left(\sigma-\sigma_{c}\right)^{-\beta}}{l\left(\sigma-\sigma_{c}\right)^{-\beta}}\right] .
$$

Figure 1 shows a data collapse according to Eq. (2) with $\beta=1.8$, in good agreement with the scaling of strain rate with the applied stress [5]. The scaling function $\mathcal{F}(x)$ exhibits a maximum and a tail, which does not contribute much to the typical escape characteristics.

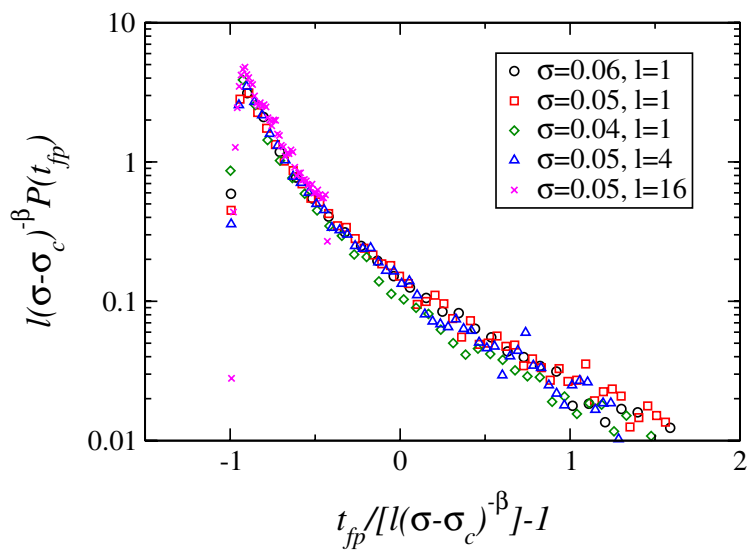

FIG. 1 (color online). Scaled first-passage time distributions according to Eq. (2), for various $\sigma$ and $l$, with $\beta=1.8$.

A cumulative version of $P\left(t_{\mathrm{fp}}\right)$ is the instantaneous selfoverlap order parameter [7] $Q_{t}(l, \tau)=1 / N \sum_{n=1}^{N} w_{n}$, where $w_{n}=1$ if the displacement of dislocation $n$ remains less than $l$ across the time interval $t \rightarrow t+\tau$ and equals 0 otherwise. The first two moments

$$
\begin{gathered}
Q(l, \tau)=\left\langle Q_{t}(l, \tau)\right\rangle, \\
\chi_{4}(l, t)=N\left[\left\langle Q_{t}(l, \tau)^{2}\right\rangle-\left\langle Q_{t}(l, \tau)\right\rangle^{2}\right]
\end{gathered}
$$

of $Q_{t}(l, \tau)$ (calculated from sample-to-sample fluctuations) are then used to characterize the dynamics. As is customary for similar problems, we consider here only samples that are still active at the observation time [14]. By construction, $Q(l, \tau)$ decays from one to zero as a function of $\tau$, while the four-point dynamic susceptibility $\chi_{4}(l, \tau)$ exhibits a peak at an intermediate $\tau=\tau_{4}^{*}$ and vanishes for both early and late times; see Fig. 2. $Q^{*}=Q\left(l, \tau_{4}^{*}\right) \approx 0.5$ is
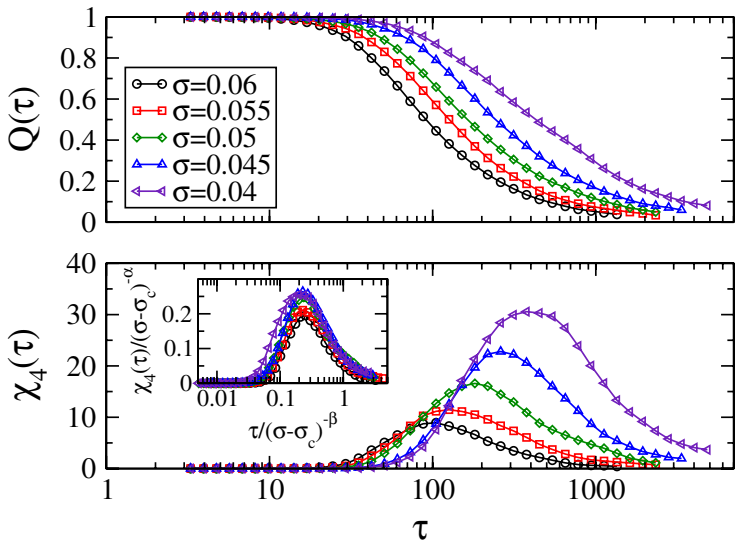

FIG. 2 (color online). Top: The average self-overlap order parameter $Q(l, \tau)$ for various values of $\sigma$ as a function $\tau$, for $l=1$. Bottom: The corresponding four-point dynamic susceptibilities $\chi_{4}(l, \tau)$. The inset shows evidence of a data collapse of $\chi_{4}(\tau)$, obtained by rescaling the data with the scaling forms of $\left\langle t_{\mathrm{fp}}\right\rangle$ and $\chi_{4}^{*}$, with $\alpha=1.1$ and $\beta=1.8$. 
nearly a constant. The peak value $\chi_{4}^{*}$ of $\chi_{4}(l, \tau)$ depends on the proximity of the critical stress $\sigma_{c}$ and has been argued to be related to the typical number of particles (here dislocations) in a correlated fast-moving domain or heterogeneity [7].

In the simulations, we observe a growing $\chi_{4}^{*}$ as $\sigma_{c}$ is approached from above, with an apparent divergence at $\sigma=\sigma_{c} \approx 0.025$; see Fig. 3. For external stresses $\sigma \leq$ 0.07 , the stress dependence of $\chi_{4}^{*}$ can be characterized by a power law $\chi_{4}^{*} \sim\left(\sigma-\sigma_{c}\right)^{-\alpha}$, with $\alpha \approx 1$.1. This result does not exhibit any significant $l$ dependence. The scaling of $\chi_{4}^{*}$ can be connected to the avalanche dynamics close to the transition by directly computing the variance of $Q(l, \tau)$ at $\tau_{4}^{*}$. Consider the distribution of avalanche sizes $s$ with a $\sigma$-dependent cutoff: $P(s)=s^{-\tau} f\left[s /\left(\sigma-\sigma_{c}\right)^{-\gamma}\right]$. By assuming that the number of dislocations $n$ swept to a distance $l$ during an avalanche obeys $\langle n\rangle \sim\langle s\rangle$ and using the condition $M\langle n\rangle=N / 2$ (arising from the observation that $Q^{*} \approx 0.5$ ) to estimate the number $M$ of independent avalanches occurring up to the delay time $\tau_{4}^{*}$, we obtain $\chi_{4}^{*}=N\left(\delta Q^{*}\right)^{2} \sim N(\sqrt{M}\langle n\rangle / N)^{2} \sim\langle n\rangle / 2 \sim(\sigma-$ $\left.\sigma_{c}\right)^{-\gamma(2-\tau)}$. As $\langle n\rangle$ grows on approaching $\sigma_{c}$, the number $M$ of heterogeneities contributing to $\chi_{4}^{*}$ decreases: An estimate for $L=300 b$ leads to $M \approx 19$ for $\sigma=0.06$ and $M \approx 6$ for $\sigma=0.037$. As the avalanche size exponent is close to the mean field $\tau \approx 1.5$ [11], $\alpha \approx 1$ would indicate $\gamma \approx 2$, again consistent with the mean-field-like scenario [15]. Incorporating a frictional stress $\sigma_{P}$ to model the Peierls barrier (relevant for bcc metals), the same results hold qualitatively true up to a $\sigma_{P}$ comparable to the yield stress $\sigma_{c}$. The yield stress for $\sigma_{P}>0$ is simply shifted such that $\hat{\sigma}_{c} \simeq \sigma_{c}+\sigma_{P}[16]$.

To further explore the possibility of identifying a divergent nonequilibrium correlation length, we consider the

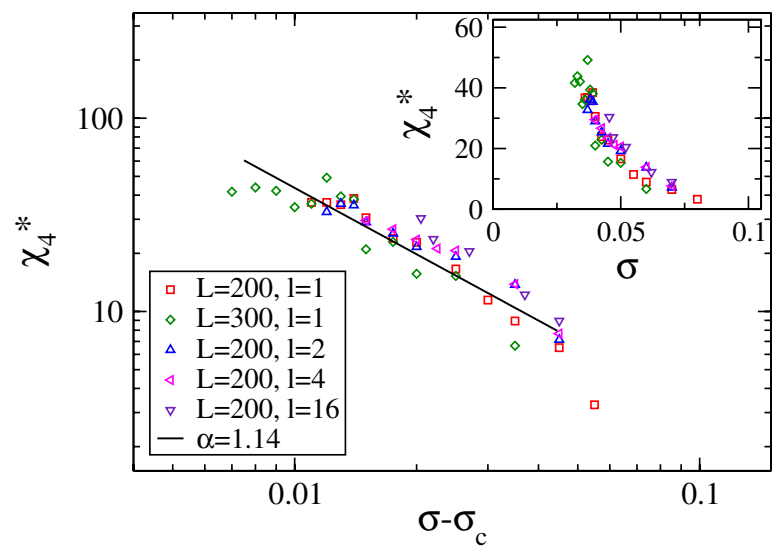

FIG. 3 (color online). The peak value $\chi_{4}^{*}$ of the dynamic susceptibility as a function of the distance from the critical stress for different system sizes $L$ and length scale parameters $l$. The solid line corresponds to a power law $\chi_{4}^{*} \sim\left(\sigma-\sigma_{c}\right)^{-\alpha}$, with $\alpha=1.14$. The inset shows the same data as a function of $\sigma$ with a linear scale. conditional probability $\quad g_{d}(\vec{r}, l)=P\left[w\left(l, \tau_{4}^{*}, \vec{r}\right)=\right.$ $\left.0 \mid w\left(l, \tau_{4}^{*}, 0\right)=0\right]$ that a dislocation at a position $\vec{r}$ has moved at least a distance $l$ up to a delay time $\tau_{4}^{*}$, given that a dislocation at the origin has done so. $g_{d}(\vec{r}, l)$ provides a measure of the spatial structure of the typical heterogeneities contributing to $\chi_{4}^{*}$. Figure 4(a) and 4(b) shows examples of this dynamical correlation function for stress values $\sigma=0.06$ and $\sigma=0.04$, respectively. We focus here for simplicity on the case $l=1$. Notice that closer to jamming, correlations clearly extend further in the $y$ direction, while we could not identify a clear trend in the $x$ direction. At the same time, the static density-density correlation function $g(\vec{r})$, computed from snapshots of the dislocation configurations in the steady state, does not show any significant dependence on $\sigma[17,18]$. To estimate the dynamical correlation length, we fitted an exponential function $\exp \left(-y / \xi_{y}\right)$ to the $g_{d}(\vec{r})$ data averaged over a narrow strip along the $y$ direction [19]. This leads to a divergent stress dependence of $\xi_{y} \sim(\sigma-$ $\left.\sigma_{c}\right)^{-\nu_{y}}$, with $\nu_{y} \approx 1$; see Fig. 5. A similar analysis in the $x$ direction is less conclusive. Because of the tendency of dislocations to form wall-like structures, the statistics is significantly worse in the $x$ direction, and no clear trend is visible for the evolution of $\xi_{x}$ with $\sigma$ (inset in Fig. 5): $\xi_{x}$ appears to have a relatively large stress-independent value around $\xi_{x} \approx 35 b$ [20]. In 3D DDD simulations [21], avalanches have been observed to be characterized by a lamellar shape, something that could be related to this large value of $\xi_{x}$. Indications of similar anisotropic avalanches have been observed in a continuum model of yielding [22].
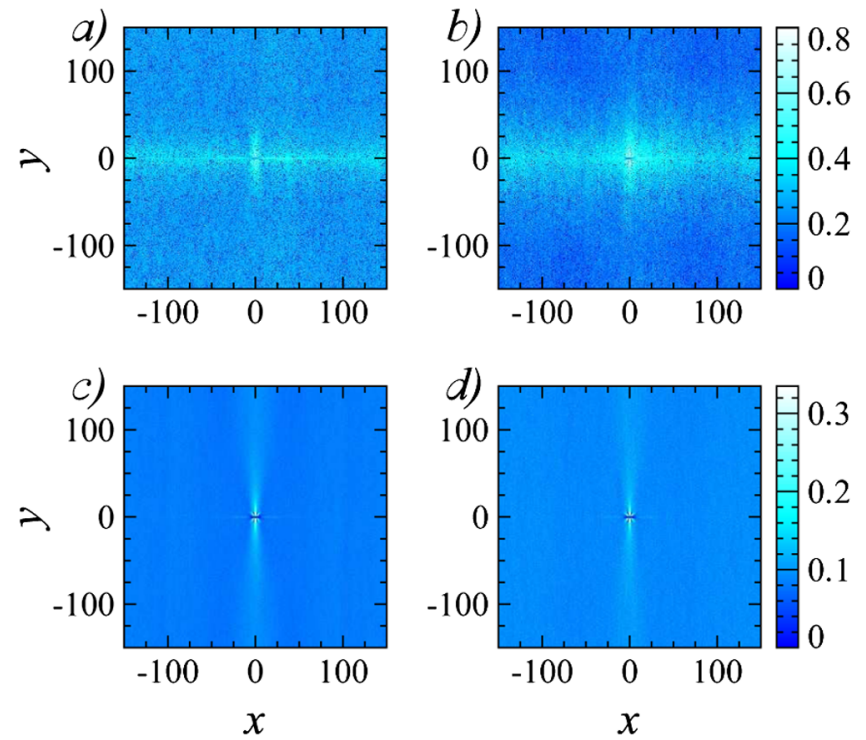

FIG. 4 (color online). The dynamic and static correlation functions $(L=300 b$ and $l=1)$. In (a) and (b) we show the dynamic correlation functions $g_{d}(\vec{r}, l=1)$ for $\sigma=0.06$ and $\sigma=0.04$, respectively. (c) and (d) show the static correlation function $g(\vec{r})$ for the same stress values $[\sigma=0.06$ in (c) and $\sigma=0.04$ in (d)]. 


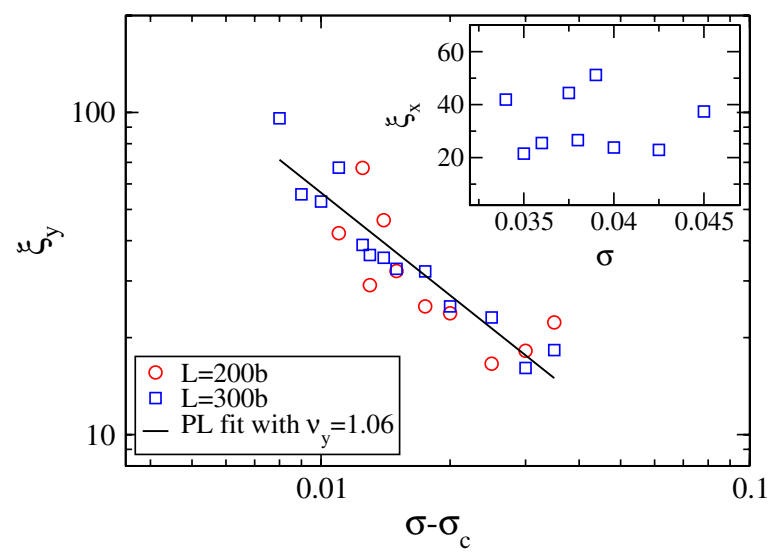

FIG. 5 (color online). Main figure: The dynamic correlation length $\xi_{y}$ in the $y$ direction, exhibiting a power law divergence close to $\sigma_{c}$. Inset: The dynamic correlation length $\xi_{x}$ in the $x$ direction. The length scale parameter $l=1$ is used.

Notice also that the observed scaling of $\xi_{y}$ is consistent with the mean field cutoff scaling of the avalanche size distribution, assuming that $\xi_{y}$ measures the linear size of a typical avalanche in the $y$ direction.

In summary, we have shown that the dislocation jamming transition is accompanied by a divergent dynamic susceptibility and a divergent correlation length. This scaling, which we relate to the avalanche dynamics of the system, is purely dynamical in nature: The static correlation function characterizing the dislocation structures does not exhibit any significant dependence on $\sigma$. The observed exponent values $\alpha \approx \nu_{y} \approx 1$ indicate that the heterogeneities are compact and characterized by a single diverging correlation length in the $y$ direction, thus clearly demonstrating that the dislocation jamming transition is indeed a true nonequilibrium second-order phase transition. It would be interesting to test similar avalanche-based ideas also for plasticity of amorphous solids [23]. Notice that, despite similarities with other relevant systems such as granular media, there are also significant differences: In the dislocation system the dynamics stops due to the formation of various metastable dislocation structures (dipoles, walls, and more complicated multipolar structures) arising from the combination of anisotropic long-range dislocation-dislocation interactions and restricted dislocation motion along a single slip direction. On the other hand, e.g., in granular systems, jamming takes place when grains get stuck by short-range contact forces within cages formed by their nearest neighbors, a process that is not accompanied by any large scale grain structures. The observation that in the present case the results seem to be independent of $l$ close enough to the transition suggests that, contrary to many other systems where $l$ needs to be tuned to a specific value related to an underlying "cage size," such a typical microscopic length does not exist here. This indicates that the same scale-free property would apply also for the force landscape experienced by the dislocations, with the first-passage properties related to the time it takes for the force landscape to deform on the observation scale, due to the avalanche dynamics.

D. J. Durian and M. Zaiser are thanked for useful comments. We acknowledge the support of the Academy of Finland (via a postdoctoral grant and via the Center of Excellence program) and the European Commissions NEST Pathfinder program TRIGS under Contract No. NEST-2005-PATH-COM-043386. M.-C. M. acknowledges the support of the Ministerio de Educación y Ciencia (Spain) under Grant No. FIS2007-66485-C02-02 and the Generalitat de Catalunya and the Ministerio de Educación y Ciencia (Programa I3).

[1] Jamming and Rheology, edited by A.J. Liu and S. R. Nagel (Taylor \& Francis, London, 2001).

[2] G. Biroli, Nature Phys. 3, 222 (2007).

[3] C. S. O'Hern et al., Phys. Rev. Lett. 88, 075507 (2002).

[4] Jamming, Yielding and Irreversible Deformation in Condensed Matter, edited by M.C. Miguel and J.M. Rubi, Lect. Notes Phys. Vol. 688 (Springer, Berlin, 2006).

[5] M. C. Miguel et al., Phys. Rev. Lett. 89, 165501 (2002).

[6] A. S. Keys et al., Nature Phys. 3, 260 (2007).

[7] A. R. Abate and D. J. Durian, Phys. Rev. E 76, 021306 (2007).

[8] L. Berthier et al., Science 310, 1797 (2005).

[9] M. C. Miguel et al., Nature (London) 410, 667 (2001).

[10] L. Nicola, E. Van der Giessen, and A. Needleman, J. Appl. Phys. 93, 5920 (2003).

[11] M.C. Miguel et al., Mater. Sci. Eng. A 309-310, 324 (2001).

[12] In Ref. [5], $\sigma_{c} \approx 0.01$, but here we employ periodic boundary conditions in both $x$ and $y$ directions, increasing $\sigma_{c}$.

[13] Because of the scale-free dislocation interactions, our results hold also for other dislocation densities.

[14] M. M. de Oliveira and R. Dickman, Phys. Rev. E 71, 016129 (2005).

[15] M. Zaiser, B. Marmo, and P. Moretti, Proc. Sci., SMPRI2005 (2005) 053.

[16] L. Laurson, M.C. Miguel, and M. J. Alava (to be published).

[17] M. Zaiser, M. C. Miguel, and I. Groma, Phys. Rev. B 64, 224102 (2001).

[18] We consider the pair correlation of dislocations irrespective of the sign of their Burgers vector, while in Ref. [17] dislocations of the same and different signs were considered.

[19] Different strip widths and averaging over a narrow angle yield similar results.

[20] Note that we use periodic boundary conditions and that $\xi_{x}$ is not much smaller than $L / 2$.

[21] F. F. Csikor et al., Science 318, 251 (2007).

[22] M. Zaiser, Adv. Phys. 55, 185 (2006).

[23] A. Lemaitre and C. Caroli, Phys. Rev. Lett. 103, 065501 (2009). 